\title{
Nonlinear Optics and Spectroscopy
}

\section{NICOLAAS BLOEMBERGEN}

Harvard University, Cambridge, Massachusetts 02138

This article is the lecture the author delivered in Stockholm on 8 December 1981 when he received the Nobel Prize in Physics, which he shared with Kai M. Siegbahn and Arthur L. Schawlow. As the article appeared in Science 216, 1057-1064 (4 June 1982), it is not reproduced here. 